\title{
Adding Playful Interaction to Public Spaces
}

\author{
Amnon Dekel1,2, Yitzhak Simon ${ }^{2.1}$, Hila Dar ${ }^{2.2}$, Ezri Tarazi ${ }^{2.2}$,Oren Rabinowitz ${ }^{2.3}$, \\ Yoav Sterman ${ }^{2}$ \\ ${ }^{1}$ The Hebrew University Jerusalem \\ amnoid@cs.huji.ac.il \\ 2 The Bezalel Academy of Art and Design, Jerusalem \\ 2.1 archsimon@013.net \\ $2.2\left\{\right.$ hila, ezri\}@bezalel.ac.il, ${ }^{2.3}$ orenin@gmail.com
}

\begin{abstract}
Public spaces are interactive by the very fact that they are designed to be looked at, walked around, and used by multitudes of people on a daily basis. Architects design such spaces to create physical scenarios for people to interact with, but this interaction will usually be one sided- the physical space does not usually change or react. In this paper we present three interaction design projects which add reactive dynamics into objects located in public spaces and in the process enhance the forms of interaction possible with them.
\end{abstract}

\section{Introduction}

Public spaces are an inseparable part of the urban fabric. Such spaces function mostly as passageways from place to place, or for short term activities. A public park, for example, where people spend relatively more time, contains a larger percentage of non built areas, which create areas for sitting, resting, and playing. A city center's squares and boulevards allocate a smaller percentage of their areas for benches and other places to hang around in, while leaving larger areas for human pedestrian travel which takes place on sidewalks and walkways. The design of these spaces will affect the types of activities that will be held in them - exposed places will encourage public interaction, whereas enclosed spaces will enable more intimate social interactions. Both of these models are important for a city since they satisfy people's basic needs for public or intimate social encounters, and even enable a form of urban escapism.

\subsection{Playful Interactivity}

We define playful interactivity as any human computer interaction that has at its core no pragmatic goals- in such situations users are more interested in enjoying themselves than they are in achieving a specific task. Using this definition, most computer games are not examples of playful interactivity since the people playing them are usually very goal oriented in their efforts to beat another player or to gain more points. A good example of playful interactivity is a music generation game such as Stella and the Star-Tones [6] in which the user explores ways to create nice sounding 
music. When playing the game, users are not competing with anyone, but are rather in a state of "doodling" with music and visuals and enjoying themselves. The process usually lasts no more than a few minutes, after which one may get bored or simply have to return to work. In the projects outlined below, the goal was to add a playful interactive layer to objects that people are used to seeing in public spaces: water falls, rows of sculptural blocks, and benches. The motivation was to see how effective such playful interactivity would be, as well as to explore how its addition will affect the way people interact with objects they already know very well. The work builds on previous work on the creation of social interaction in public spaces by Yoon et. al. [8] and Rogers \& Brignull [1], but differs in that it does not necessitate the use of high cost projection devices.

\section{The Sonic Waterfall}

The Sonic Waterfall (designed and built by Yitzhak Simon) explores the effect of adding interactive aural elements to a public waterfall. A prototype was designed and built which included a pumped water system running over an ultrasonic sensor system aimed parallel to the water. As visitors ran their hands through the water they broke one of the hidden wireless sensing circuits which activated a sensor. An attached microcontroller (MC) communicated which sensor was activated to a nearby workstation. An application on the workstation parsed and used this data and combined it with a decision algorithm to play sounds or music in the vicinity of the waterfall.

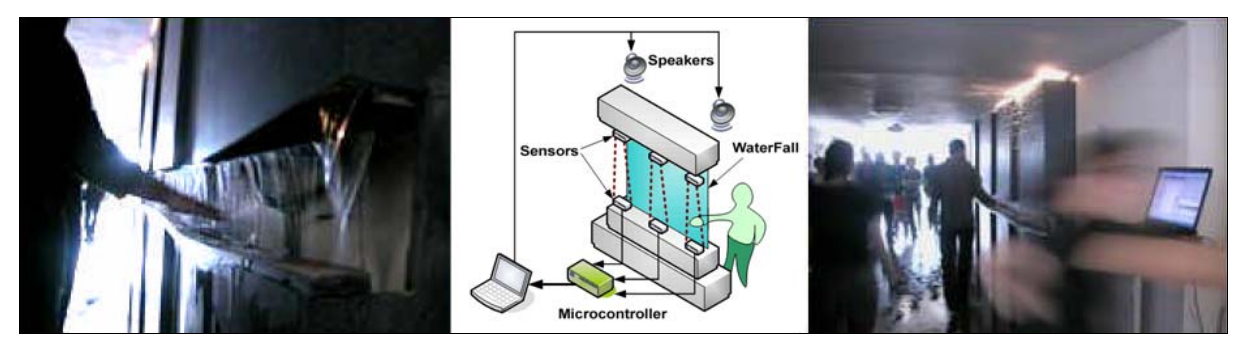

Fig. 1. The Sonic Waterfall:

The sonic waterfall was installed in one of the entrance corridors of the Bezalel Academy of Art and Design. Our observations found that the interactive audio element caused the waterfall to be extremely enticing and resulted in people standing to watch, waiting for their turn to play. One shortcoming we quickly noticed was that only one person could use the waterfall at a time if the desired outcome was to be aesthetically pleasing music. When we used non-musical audio, a more freeform interaction could take place, and more than one person could interact without downgrading the aural experience of the piece. 


\section{Musical Chairs}

The musical chairs project explores the addition of aural and visual interaction into a group of blocks set in a public space. In this project, designed and built by Yitzhak Simon and Oren Rabinowitz, visitors see a row of stool high blocks. Sitting on a block creates a short audio-visual reaction. The real action starts when someone sits on another bloc; when that happens all the blocks in between erupt in a wave of audio-visual action. This wave creates a visual animation effect accompanied by sounds which together cause a musical and playful effect. If a "circuit" is cut by someone sitting on a seat in between the current ones being occupied, the wave now moves between the first seat and the new seat. The second seat is now left out of the loop, forcing that person to move to another block in order to get back into the action.

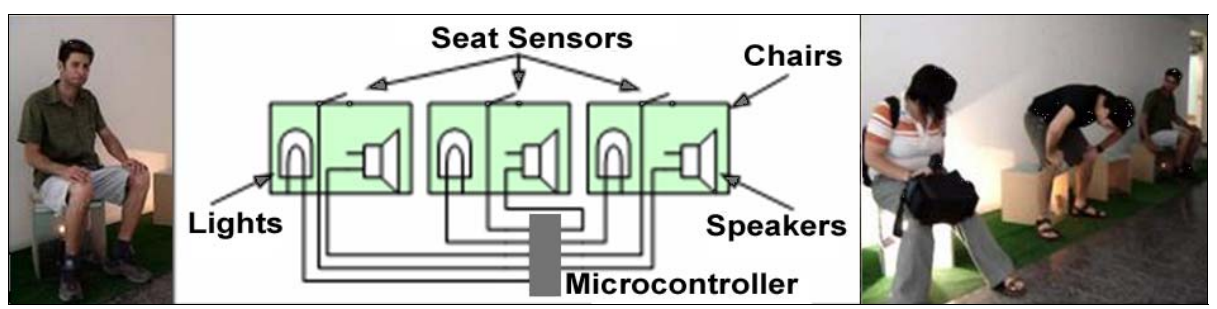

Fig. 2. Musical Chairs:

Our observations show that when people first sit on a block, they are surprised by the audio-visual reaction, but subsequently start exploring by moving to another seat to cause a reaction. At this point a second person usually joins in, sitting on a second block in unison. The ensuing audio visual "wave" surprises the participants and causes them to start exploring. One of them gets up, then the other, and then they both sit down or get up together. In this process they learn the vocabulary of the system while enjoying themselves. While they are doing this, a crowd forms around them and then a third person joins the fun, cutting off the wave in the middle and exploring further options.

\section{The Intimate Bench}

One of the most ubiquitous elements in any public space is the bench. People sit on them to read the morning newspaper, watch their children play, eat a sandwich, wait for someone, and even to sleep. An additional scenario that sometimes takes place on the bench is that of a couple who use it as a semi-intimate place to be together. The Intimate Bench project, designed and built by Yoav Sterman, is meant to explore this social scenario.

The bench (seen in figure 3 below) looks and feels like an ordinary park bench, but embedded into it are seat sensors which are connected to an embedded MC. When someone sits on the bench it reacts by turning on embedded lights in various configurations which are sensitive to the position of the people sitting on it. Thus, when peo- 
ple sit far from each other (on both ends), it reacts in ways which try to get them to communicate and come closer together. When they are close together the Bench reacts in ways that are meant to strengthen the situation. The lights in the bench cause visual shapes (hearts and triangles in red and yellow) to appear, with the hope that the shapes will subtly encroach into the participants interaction (if at first by surprise and delight, then by humor and a breaking of the social "ice"). The prototype chair has not been tested yet in public, but initial presentations found that onlookers seemed very enticed by the sudden appearance of shapes in the actual wood (which until then were completely invisible). Further testing is planned.

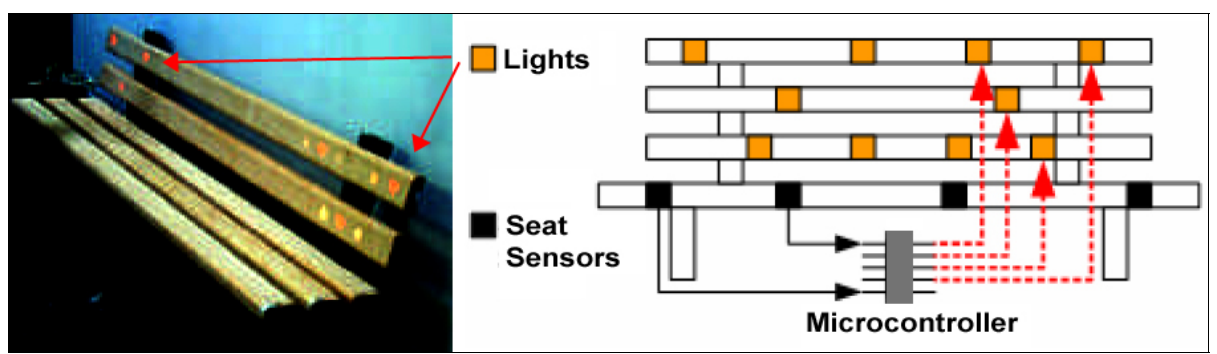

Fig. 3.The Intimate Bench:

Left: Photo of the Bench with lights on. Right: System Schematic.

\section{Conclusions and Summary}

This paper presented design projects in the Texperience (Technology for Experience) course $^{1}$ given at the Bezalel Academy of Art and Design in Jerusalem in the Master of Design program in Industrial Design. The main goal of these projects was to add a playful interactive layer to objects that people are used to seeing in public spaces. Our motivation was to see how effective such playful interactivity would be next to exploring how its addition will affect the way people view and interact with objects they already know very well. Our observations brought us to the realization that as long as the interactive vocabulary of such systems is simple enough to be understood quickly by viewers and participants, then they can be effective. This is a clear barrier- a system that is too complicated to understand within a 30 second window will go unused in most cases. We view effectiveness in this context as a situation in which casual participants, who do not have much time on their hands, will successfully negotiate and understand the interaction model of the object and thus enter into an enjoyable and playful session with it.

We also understood that the content and dynamics of the reactive objects must be designed to take all the possible interaction scenarios into consideration in order to be successful. As we saw in the Sonic Waterfall project, the successful creation of music was only possible when done by a single participant. A simple change in the sounds generated by the system (from music to sound effects) enabled multiple users to generate a pleasing sonic texture.

\footnotetext{
${ }^{1}$ The course was developed and taught by Amnon Dekel, Hila Dar and Ezri Tarazi
} 
As for how the addition of interactivity affects the way people view and interact with well known objects, we observed that the objects, that in many cases have become almost invisible in the background, suddenly gained peoples' attention. People could not walk by without at least noticing that something unique and interesting was happening, and in many cases they stood around to get a chance to interact with the objects. In some ways we could say that adding interactivity to such objects can bring them back to life, although we by no means should be understood as stating that interactivity should be added everywhere. All we hope to achieve is the opening of a discourse centered on the place of interactivity in the design of public spaces.

Are such objects ready for prime time? We feel that more research and exploration must be done to explore how such interaction models will weather the passage of time- will they age elegantly, and take their place as a new form of public object? Or will they become aural and visual noise generators that annoy passers by, ultimately suffering damage by angry onlookers or turned off by city officials. Only field tests and time will tell.

\section{References}

1. Brignull, H, Rogers, Y.: Enticing people to interact with large public displays in public spaces. INTERACT'03. M. Rauterberg et al. (Eds.) Published by IOS Press, (c) IFIP, 2003, pp. 17-24

2. Carmona, M., Heath, T., Oc, T., Tiesdel, S: Public places - Urban Spaces, The dimensions of Urban Design, Architectural Press 2003

3. Lebesch, B., Sherman,C., Williams, G.: Stella and the Star-tones, Bohem Interactive, 1996

4. Paradiso, J. New Sensor Architectures for Responsive Environments. Talk given at Perdue University on October 13, 2004. See online PDF at [http://www.iee.org/OnComms/PN/controlauto/Paradiso\%20paper.pdf]

5. Paradiso, J.A., Hsiao, K., Strickon, J., Lifton, J. and Adler, A., "Sensor Systems for Interactive Surfaces,” IBM Systems Journal, Vol. 39, No. 3\&4, October 2000, pp. 892- 914

6. Stuart Reeves, Steve Benford, Claire O'Malley, Mike Fraser: Public life: Designing the spectator experience. Proceedings of the SIGCHI conference on Human factors in computing systems. 2005

7. Van Kleek, M: Intelligent Environments for Informal Public Spaces: the Ki/o Kiosk Platform. MIT Masters thesis, 2003.

8. Jennifer Yoon, Jun Oishi, Jason Nawyn, Kazue Kobayashi, Neeti Gupta.

FishPong: Encouraging Human-to-Human Interaction in Informal Social Environments, CSCW’04, November 6-10, 2004 